Research Article

\title{
Fractional-Order Control of a Micrometric Linear Axis
}

\author{
Luca Bruzzone and Pietro Fanghella \\ DIME, Department of Mechanical, Energetic, Management and Transport Engineering, University of Genova, \\ via Opera Pia 15A, 16145 Genova, Italy
}

Correspondence should be addressed to Luca Bruzzone; bruzzone@dimec.unige.it

Received 13 December 2012; Accepted 18 February 2013

Academic Editor: Mohamed Zribi

Copyright (C) 2013 L. Bruzzone and P. Fanghella. This is an open access article distributed under the Creative Commons Attribution License, which permits unrestricted use, distribution, and reproduction in any medium, provided the original work is properly cited.

This paper discusses the application of a particular fractional-order control scheme, the PDD ${ }^{1 / 2}$, to the position control of a micrometric linear axis. The $\mathrm{PDD}^{1 / 2}$ scheme derives from the classical PD scheme with the introduction of the half-derivative term. The $\mathrm{PD}$ and $\mathrm{PDD}^{1 / 2}$ schemes are compared by adopting a nondimensional approach for the sake of generality. The linear model of the closed-loop system is discussed by analysing the pole location in the $\sigma$-plane. Then, different combinations of the derivative and half-derivative terms, characterized by the same settling energy in the step response, are experimentally compared in the real mechatronic application, with nonnegligible friction effects and a position set point with trapezoidal speed law. The experimental results are coherent with the nonlinear model of the controlled system and confirm that the introduction of the half-derivative term is an interesting option for reducing the tracking error in the transient state.

\section{Introduction}

Fractional calculus is a branch of classical mathematics which considers derivatives and integrals to be of a noninteger order [1-3]. The origin of fractional calculus dates back to the seventeenth century; it was discussed by Leibniz, De L'Hospital, Euler, Fourier, Liouville, and Riemann; nevertheless, its practical applications are relatively recent.

In general, natural and artificial phenomena can be properly modelled by means of integer-order (IO) differential equations, even if many physical variational principles, such as the Euler-Lagrange equations, the Hamilton equations, and the Dirac equations, can be expressed by fractional-order (FO) formulations [4-6].

On the other hand, some real systems can be modelled adequately only by means of FO differential equations; in particular, fractional calculus is a powerful tool in analysing multiscale problems, characterized by wide time or length scales; for example, FO differential equations model properly dielectrics and viscoelastic materials over extended ranges of time and frequency [7-9]; in heat transfer and electrochemistry, the half-order fractional integral is the natural integral operator that connects the thermal or material gradients with the heat diffusion $[10,11]$. Other applications of Fractional
Calculus in physics are discussed in $[12,13]$, and a physical interpretation of fractional derivatives is outlined in [14].

Fractional calculus can be exploited not only for modelling physical phenomena, but also in engineering applications, such as electronics, signal processing, and bioengineering $[15,16]$; in particular, Fractional Calculus is a powerful tool in the area of control system design [17-20].

In a closed-loop system, both the plant and the controller can be of fractional order or integeral order; therefore, four possible cases are possible: IO plant with IO controller, IO plant with FO controller, FO plant with IO controller, and FO plant with FO controller; however, since FO plants are far more infrequent than IO plants, it is interesting to focus the attention on the comparison between IO controllers and FO controllers for IO plants.

The most common approach to FO control design is represented by the $\mathrm{PI}^{\lambda} \mathrm{D}^{\mu}$ scheme, which generalizes the PID scheme, characterized by the proportional, first-order integral, and first-order derivative terms, by adopting derivatives and integrals of nonintegeral order $\lambda$ and $\mu$; these orders are additional parameters that can be tuned to optimize the closed-loop system behaviour [21]. Design techniques and methods for the synthesis of $\mathrm{PI}^{\lambda} \mathrm{D}^{\mu}$ controllers are discussed in [22-24], and an optimization tool for tuning $\mathrm{PI}^{\lambda} \mathrm{D}^{\mu}$ 
controls starting from given specifications is presented in [25]. In [26], a Model Algorithmic Controller with $\mathrm{PI}^{\lambda} \mathrm{D}^{\mu}$ structure is proposed, which combines the benefits of both the fractional-order PID and MAC schemes. The disturbance rejection problem for fractional-order PID controllers is discussed in $[27,28]$; in particular, in $[28]$ the $\mathrm{PI}^{\lambda} \mathrm{D}^{\mu}$ scheme is used in combination with a neural network and a finite-impulse-response-type representation. The practical implementation of a fractional-order controller for a DC motor is discussed in [29].

An approach to fractional-order control which is alternative to the $\mathrm{PI}^{\lambda} \mathrm{D}^{\mu}$ scheme is proposed in $[30,31]$, where the $\mathrm{PDD}^{1 / 2}$ scheme is introduced and compared to the classical PD scheme in the control of mechanical systems. In the $\mathrm{PDD}^{1 / 2}$ control scheme, besides the proportional term $(\mathrm{P})$ and the derivative term $(\mathrm{D})$, also the half-derivative term $\left(D^{1 / 2}\right)$, which is proportional to the derivative of order $1 / 2$ of the error, is exploited; therefore, instead of replacing the first-order derivative with a FO derivative, the first-order derivative term and the half-derivative term are used in combination. The origin of the this scheme is the idea that control system designers are unlikely to abandon the well-known $\mathrm{PID} / \mathrm{PD}$ scheme, and the addition of the half-derivative term might be more acceptable than the replacement of the firstorder derivative term with a fractional-order derivative term (the integral term of the PID, which eliminates the steadystate error, is not considered in this comparison, which is focussed on the transient state).

In [32], the PD and $\mathrm{PDD}^{1 / 2}$ controls of a purely inertial system are compared, adopting a nondimensional approach for the sake of generality and introducing the dimensionless settling energy, which represents the effort of the control system to drive the controlled system to steady state. Different combinations of the derivative and half-derivative terms are compared by simulation, keeping this parameter in the step response constant. The simulation results show that using both terms in combination $\left(\mathrm{PDD}^{1 / 2}\right)$ improves the control performance in terms of settling time and rise time with respect to the pure $\mathrm{PD}$ and pure $\mathrm{PD}^{1 / 2}$ schemes. This is a clear indication that the $\mathrm{PDD}^{1 / 2}$ can introduce benefits with respect to the classical fractional-order extension of the PD scheme, that is, the $\mathrm{PD}^{\mu}$ scheme.

The contents of the paper are as follows.

(i) Section 2 discusses the Grünwald-Letnikov definition of FO derivative and its discrete-time approximation, which is adopted in the rest of the work.

(ii) Section 3 recalls the stability conditions for FO systems.

(iii) Sections 4 and 5 extend the theoretical results of [32] with a discussion about the pole location and the stability of the a purely inertial system with $\mathrm{PDD}^{1 / 2}$ control.

(iv) In Section 6, the theoretical and simulation results of Sections 4 and 5 are validated by applying the PD and $\mathrm{PDD}^{1 / 2}$ schemes to the position control of a highperformance micrometric linear axis, actuated by a brushless linear DC motor; a nonlinear model of the system, including friction effects, is developed; the experimental results are coherent with the simulations on the nonlinear model and confirm the benefits of the $\mathrm{PDD}^{1 / 2}$ control scheme.

(v) Section 7 outlines the conclusions.

\section{Definition and Numerical Evaluation of Fractional-Order Derivatives}

In Fractional calculus, both integration and differentiation to a noninteger order are expressed by the continuous integrodifferential operator ${ }_{a} D_{t}^{\alpha}$, which is defined as

$$
{ }_{a} D_{t}^{\alpha}= \begin{cases}\frac{d^{\alpha}}{d t^{\alpha}} & \operatorname{Re}(\alpha)>0, \\ 1 & \operatorname{Re}(\alpha)=0, \\ \int_{a}^{t}(d \tau)^{-\alpha} & \operatorname{Re}(\alpha)<0,\end{cases}
$$

where $a$ and $t$ are the limits of the operation, and $\alpha$ is the order, which can be even complex. In the scientific literature, there are different definitions of this operator (GrünwaldLetnikov, Riemann-Liouville, Tustin, Simpson, and Caputo among others), but fortunately all of these are proved to be equivalent. In the following, we will use the GrünwaldLetnikov definition, which is most frequently adopted in control synthesis because it leads to a robust discrete-time implementation [33].

According to the Grünwald-Letnikov definition, the derivative of fractional order $\alpha$ of a function of time $x(t)$ is the following:

$$
\begin{aligned}
{ }_{a} & D_{t}^{\alpha} x(t) \\
& =\lim _{h \rightarrow 0}\left[\frac{1}{h^{\alpha}} \sum_{k=0}^{[(t-a) / h]}(-1)^{k} \frac{\Gamma(\alpha+1)}{\Gamma(k+1) \Gamma(\alpha-k+1)} x(t-k h)\right],
\end{aligned}
$$

where $h$ is the time increment, and $\Gamma$ is the Gamma function, which extends the factorial function to real and complex numbers and is defined by the following equation:

$$
\Gamma(z)=\int_{0}^{\infty} t^{z-1} e^{-t} d t
$$

In (2), there is the sum of an infinite number of terms, as $h$ tends to zero; in order to carry out a numerical computation, (2) can be rewritten adopting a small but finite sampling time $T_{s}$, in order to obtain the following discretetime approximation [34]:

$$
{ }_{a} D_{t}^{\alpha} x(t) \cong{ }_{a} D^{\alpha} x_{k}=\left[\frac{1}{T_{s}^{\alpha}} \sum_{j=0}^{k} w_{j}^{\alpha} x\left(t-j T_{s}\right)\right],
$$

where $k=(t-a) / T_{s}$ is the current step, and

$$
\begin{aligned}
& w_{0}^{\alpha}=1, \\
& w_{j}^{\alpha}=\left(1-\frac{\alpha+1}{j}\right) w_{j-1}^{\alpha}, \quad j=1,2, \ldots .
\end{aligned}
$$




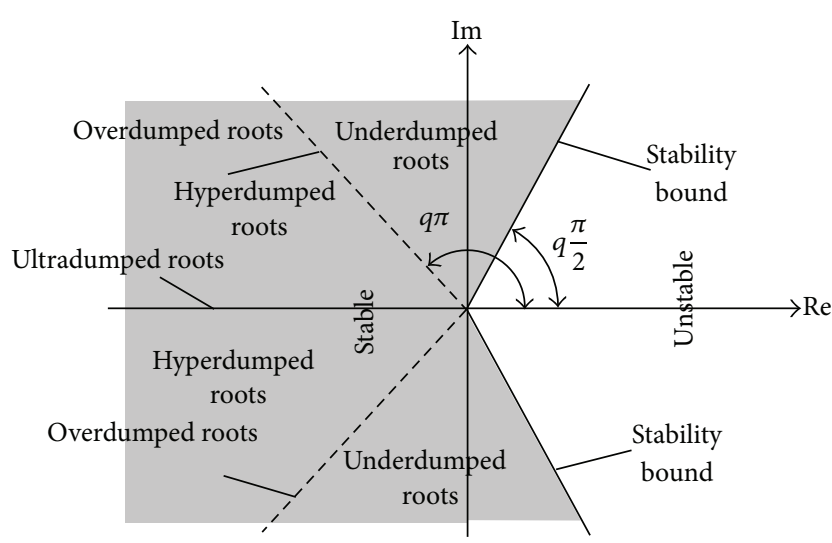

FIGURE 1: Stability regions of fractional-order systems with fractional commensurate order $q$.

\section{Stability of Fractional-Order Systems}

The Laplace transform can be applied to fractional-order derivatives of a given signal; similarly to integer-order derivatives, if the function of time $x(t)$ and its derivatives at $t=0$ are all equal to zero, it is possible to demonstrate that [34]:

$$
\mathscr{L}\left[D^{\alpha} x(t)\right]=s^{\alpha} \mathscr{L}[x(t)] .
$$

According to the Matignon stability theorem [35], a fractional transfer function $G(s)=Z(s) / P(s)$ of a linear time-invariant system is stable if and only if the following condition is satisfied in $\sigma$-plane:

$$
|\arg (\sigma)|>q \frac{\pi}{2}, \quad \forall \sigma \in C, P(\sigma)=0,
$$

where $\sigma=s^{q}$ and $q$ is the fractional commensurate order ( $q=1 / 2$ for a closed-loop system with IO plant and $\mathrm{PDD}^{1 / 2}$ control [35]). When $s=0$ is a single root of $P(s)$, the system cannot be stable.

For $q=1$ (IO systems), this theorem defines the classical requirement of pole location in the complex plane; for stability, no pole must be in the closed right half-plane, and the stability boundary in the $s$-plane is the imaginary axis.

Moreover, it is possible to demonstrate that, for FO systems, in the $\sigma$-plane [36] (see Figure 1):

(i) the region with $q(\pi / 2)<|\arg (\sigma)|<q \pi$ corresponds to stable underdamped behaviour;

(ii) the pair of lines at $|\arg (\sigma)|=q \pi$ correspond to stable overdamped behaviour;

(iii) the region with $q \pi<|\arg (\sigma)|<\pi$ corresponds to stable hyperdamped behaviour;

(iv) the negative real axis $(|\arg (\sigma)|=\pi)$ corresponds to stable ultradamped behaviour.

Within the stability region, the time response is oscillatory if there are roots in the underdamped region.

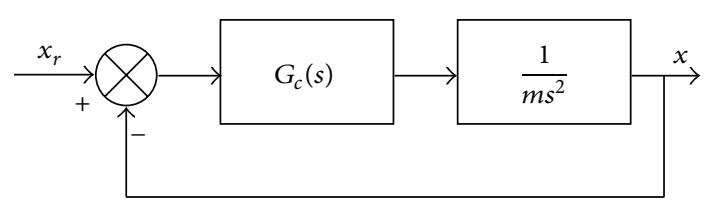

FIGURE 2: Block scheme of the closed-loop system.

\section{Purely Inertial System with PDD $^{1 / 2}$ Control: Linear Model and Stability}

Figure 2 shows the block scheme of the considered model, a second-order linear translational system with mass $m$, controlled by a $\mathrm{PDD}^{1 / 2}$ control system with the following transfer function:

$$
G_{c}(s)=K_{p}+K_{d} s+K_{h d} s^{1 / 2},
$$

where $K_{p}$ is the proportional gain, $K_{d}$ is the derivative gain, and $K_{h d}$ is the half-derivative gain.

The fractional-order differential equation of the whole system is

$$
m \frac{d^{2}}{d t^{2}} x=K_{p} e_{x}+K_{d} \frac{d}{d t} e_{x}+K_{h d} \frac{d^{1 / 2}}{d t^{1 / 2}} e_{x},
$$

where $x$ is the axis position and the error $e_{x}=x_{r}-x$ is the difference between the set points $x_{r}$ and $x$. For the sake of generality, the system behaviour is analysed using a dimensionless approach, introducing the dimensionless parameters $\zeta$ and $\psi[31]$ :

$$
\begin{gathered}
\zeta=\frac{K_{d}}{2 \sqrt{m K_{p}}}, \\
\psi=\frac{K_{h d}}{K_{p}} \omega_{n}^{1 / 2}=\frac{K_{h d}}{K_{p}^{3 / 4} m^{1 / 4}},
\end{gathered}
$$

where $\omega_{n}=\left(K_{p} / m\right)^{1 / 2}$ is the natural frequency of the dynamic system.

The parameter $\zeta$ represents nondimensionally the derivative gain $K_{d}$ and corresponds to the damping ratio of the second-order mechanical systems, considering the equivalences elastic force-proportional term and damping forcederivative term; on the other hand, $\psi$ represents nondimensionally the half-derivative gain $K_{h d}$.

Moreover, let us introduce the dimensionless time $t_{a d}=$ $\omega_{n} t$, the dimensionless position $x_{a d}=x / x_{r}$, and the dimensionless error $e_{x, a d}=e_{x} / x_{r}$; using these dimensionless variables, (9) becomes

$$
\frac{d^{2}}{d t_{a d}^{2}} x_{a d}=2 \zeta \frac{d}{d t_{a d}} e_{x, a d}+\psi \frac{d^{1 / 2}}{d t_{a d}^{1 / 2}} e_{x, a d}+e_{x, a d} .
$$

Replacing (9) with (11) corresponds to replacing the system of Figure 2 with the one of Figure 3, where $F_{a d}$ is the dimensionless force, $x_{r, a d}$ is the dimensionless set point, and the dimensionless transfer function of the $\mathrm{PDD}^{1 / 2}$ controller is

$$
G_{c, a d}(s)=1+\psi s^{1 / 2}+2 \zeta s .
$$




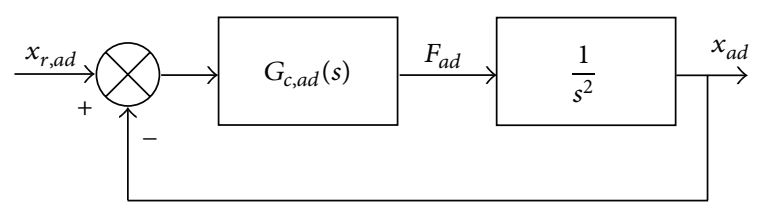

FIGURE 3: Block scheme of the dimensionless closed-loop system.

The closed-loop transfer function of the dimensionless system can be obtained by (12):

$$
\frac{x_{a d}}{x_{r, a d}}=G_{c l, a d}(s)=\frac{1+\psi s^{1 / 2}+2 \zeta s}{1+\psi s^{1 / 2}+2 \zeta s+s^{2}} .
$$

Using this approach, the system behaviour depends only on the two dimensionless parameters $\zeta$ and $\psi$.

Since the denominator of the transfer function is of second-order with commensurate order $q=1 / 2$, the system has four poles in the $\sigma$-plane. Figure 4 shows the four poles of the system for the following values of $\zeta$ and $\psi$ :

(i) $\zeta=0,0.2,0.5,0.8,1.2,1.6,2,3,4,6,8,10,12,15,20,30$;

(ii) $\psi=0,0.25,0.5,1,2,3,6,10,15,20,30,40,60$.

In Figure 4, the coloured lines connect the poles of the different systems with constant $\psi$ and variable $\zeta$, and the four colours correspond to the four poles of each system $\left(\sigma_{1}\right.$ : red; $\sigma_{2}$ : green; $\sigma_{3}$ : cyan; $\sigma_{4}$ : magenta); in the details of the $\sigma$-plane of Figures 5 and 6 , the $i$ th pole of the system characterized by $\zeta$ and $\psi$ is named $\sigma_{i}(\zeta, \psi)$.

According to the scheme of Figure 1, since $q=1 / 2$, the boundary between the oscillatory behaviour and the nonoscillatory behaviour in the time domain is the imaginary axis; observing the pole location, it is possible to note that

(i) all the systems have the poles $\sigma_{3}$ and $\sigma_{4}$ in the left half plane, negative real or complex conjugate, but these poles do not correspond to oscillatory behaviour;

(ii) all the systems have two conjugate complex poles $\sigma_{1}$ and $\sigma_{2}$ in the underdamped region $(\pi / 4<|\arg (\sigma)|<$ $\pi / 2$ ), except the systems with $\psi=0$ and $\zeta>1$; for these systems, the poles are placed on the imaginary axis, that for $q=1 / 2$ is the locus of the poles of the overdamped systems; this is coherent with the classical control theory;

(iii) consequently, the systems with $\psi>0$ and the systems with $\psi=0$ and $\zeta<1$ have oscillatory behaviour;

(iv) the system with $\psi=0$ and $\zeta=0$ is on the stability boundary $(|\arg (\sigma)|=\pi / 4)$ and has persistent oscillations in the time response, coherently with the classical control theory.

The locations of all poles indicate that the closed-loop system is always stable if at least one of the two parameters $\zeta$ and $\psi$ is greater than zero, and that the introduction of the halfderivative term exalts the oscillatory behaviour of the closedloop system.

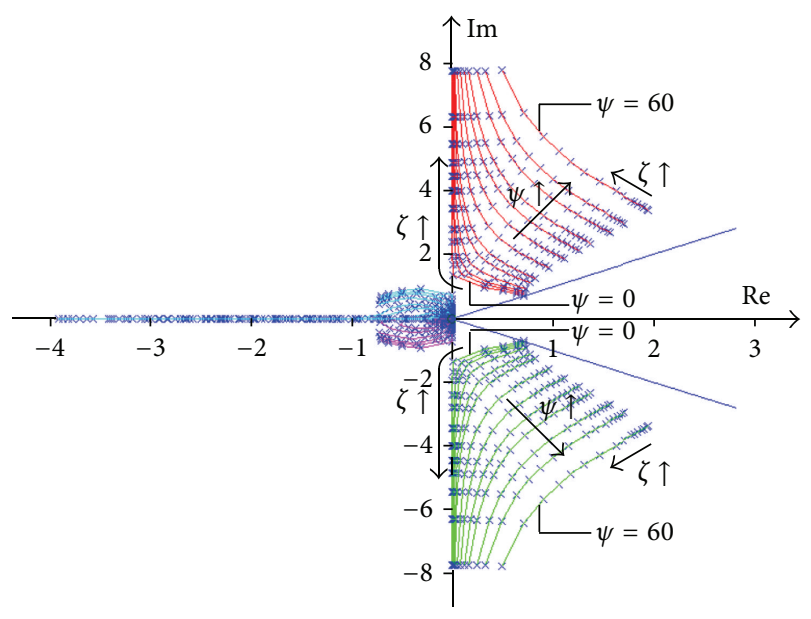

Figure 4: Poles of the closed-loop system in the $\sigma$-plane.

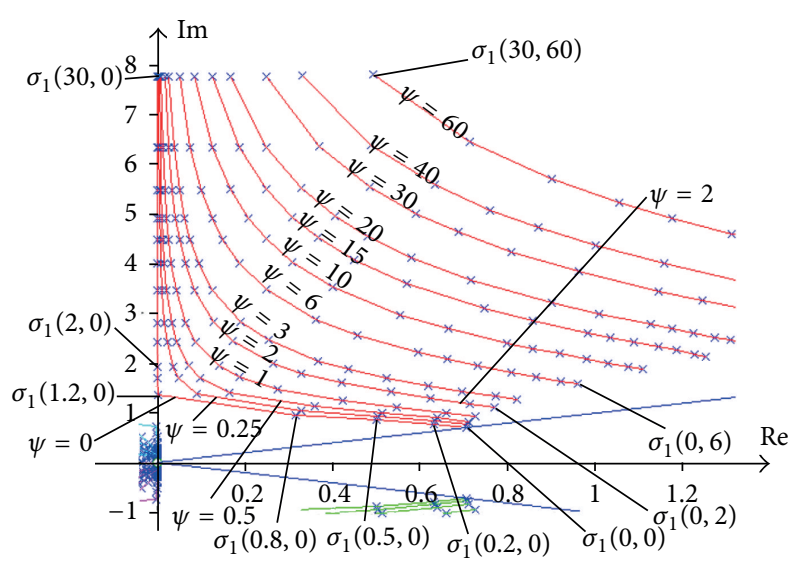

FIGURE 5: Poles of the closed-loop system in the $\sigma$-plane: details of the stable underdamped region.

\section{Combination of the Derivative and Half-Derivative Terms}

The combined effects of the derivative and half-derivative terms on the dimensionless closed-loop system are discussed in [32] with reference to the step response by means of numerical simulation, using the object-oriented Matlab library named FOTF [34]; in order to perform a systematic comparison, the dimensionless settling energy is defined according to the following equation:

$$
E_{s, a d}=\int_{0}^{\infty} F_{a d}^{2} d t_{a d} .
$$

The simulation results show that it is possible to reduce both the settling time and the rise time while maintaining the settling energy constant by using the derivative and halfderivative terms in combination. Figure 7 compares the time histories of $x_{a d}$ in the step response for the seven parameter sets of Table 1 , characterized by the same settling energy; these parameter sets have been selected evaluating the settling energy with $\zeta=1.2$ and $\psi=0$ (pure PD control), and then finding the values of $\psi$ that determine the same settling 


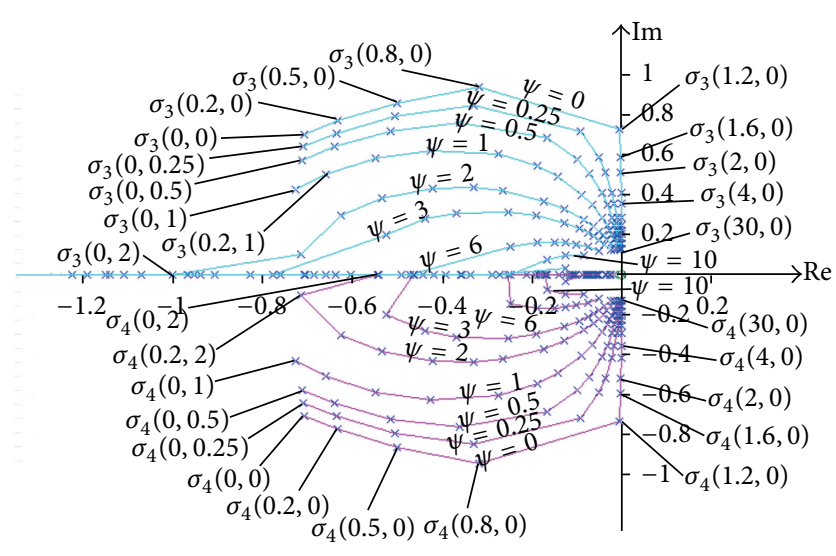

FIGURE 6: Poles of the closed-loop system in the $\sigma$-plane: details of the stable overdamped, hyperdamped, and ultradamped regions.

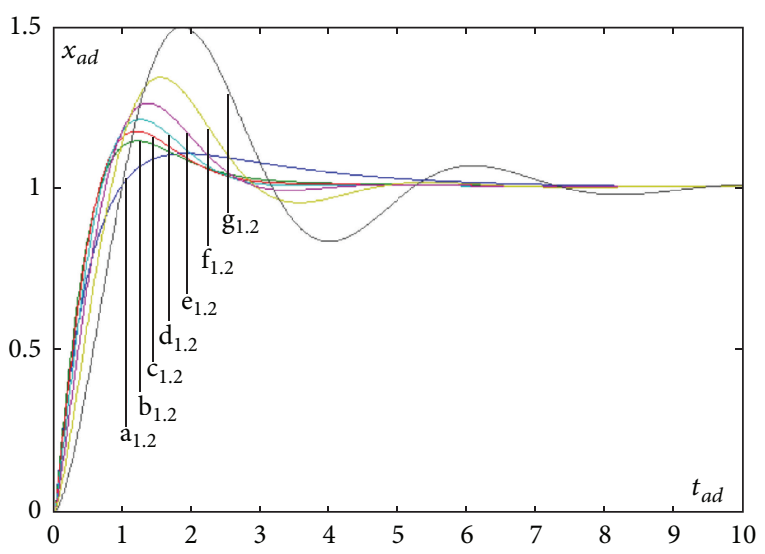

Figure 7: $x_{a d}$ as function of $t_{a d}$, parameter sets of Table 1.

energy with decreasing values of $\zeta$. Similarly, Figures 8 and 9 compare $x_{a d}$ for two groups of parameter sets (Tables 2 and $3)$, with settling energy equivalent to the PD controls with $\zeta=1$ and $\zeta=0.8$; for these two sets, differently from the case corresponding to $\zeta=1.2$, it is not possible to find a pure $\mathrm{PD}^{1 / 2}$ control with equivalent settling energy because the settling energy of the pure $\mathrm{PD}^{1 / 2}$ is higher for any value of $\psi$.

Tables 1 to 3 compare the dimensionless settling times, the dimensionless rise times, and the overshoots for the three groups of parameter sets; it is possible to note that for each group:

(i) the overshoot is lower for higher values of $\zeta$;

(ii) the settling time has a minimum: with high $\zeta$ settling is delayed by the high damping, but with low $\zeta$ settling is slowed down by the oscillations;

(iii) also the rise time has a minimum; however, the rise time has lower variations with respect to the settling time.

In general, increasing values of the half-derivative term determine a more oscillatory behaviour, and this is coherent with the pole locations discussed in Section 4.

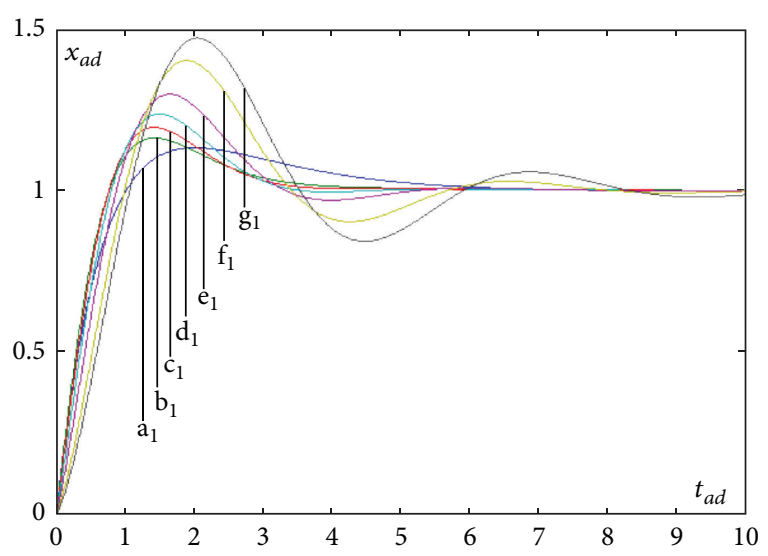

FIGURE 8: $x_{a d}$ as function of $t_{a d}$, parameter sets of Table 2.

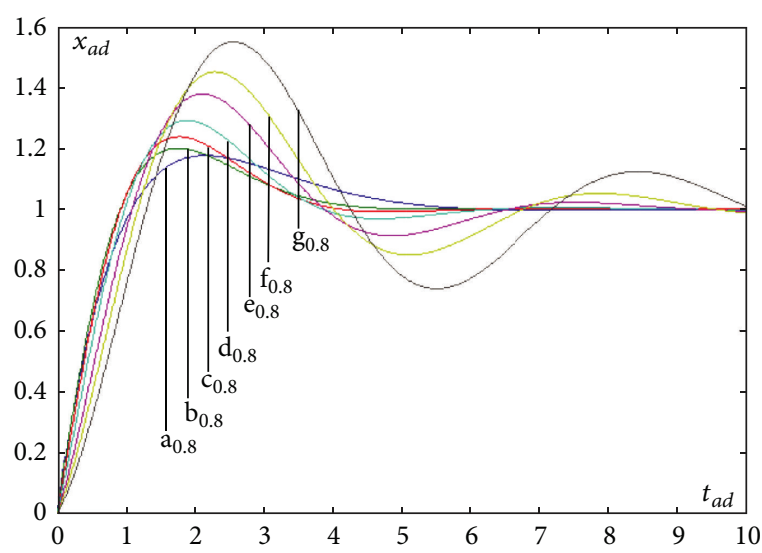

FIGURE 9: $x_{a d}$ as function of $t_{a d}$, parameter sets of Table 3 .

For each of the three levels of settling energy, it is possible to detect the $\zeta-\psi$ combination which minimizes the settling time; these combinations are reported in Table 4.

Figure 10 shows the pole locations corresponding to the six cases (three $\mathrm{PD}$ and three $\mathrm{PDD}^{1 / 2}$ ) of Table 4 ; it is possible to note that for the pure $\mathrm{PD}(\psi=0$; therefore, the system is of integeral order):

(i) if $\zeta>1$, there are 4 imaginary poles in the $\sigma$-plane, corresponding to 2 negative real poles in the $s$-plane; the system behaviour is not oscillatory;

(ii) if $\zeta=1$, there are 2 double imaginary poles in the $\sigma$-plane, $+i$ and $-i$, corresponding to the double negative real pole -1 in the $s$-plane; the system behaviour is not oscillatory;

(iii) if $\zeta<1$, there are 4 complex poles in the $\sigma$ plane, placed symmetrically with respect to both axes, corresponding to two conjugate complex roots with negative real part in the $s$-plane; the system behaviour is oscillatory.

On the other hand, for the $\mathrm{PDD}^{1 / 2}$ there are always 4 complex conjugate poles in the $\sigma$-plane, symmetric only with respect to the real axis, two in the left half-plane and two in the 
TABLE 1: $\zeta-\psi$ combinations with dimensionless settling energy equivalent to the pure PD control with $\zeta=1.2$.

\begin{tabular}{lccccc}
\hline Parameter set & $\zeta$ & $\psi$ & Overshoot $(\%)$ & Dimensionless settling time & Dimensionless rise time \\
\hline $\mathrm{a}_{1.2}$ & 1.2 & 0 & 10.42 & 5.61 & 0.67 \\
$\mathrm{~b}_{1.2}$ & 1 & 1.851 & 14.34 & 3.26 & 0.48 \\
$\mathrm{c}_{1.2}$ & 0.8 & 2.193 & 17.39 & 2.87 & 0.48 \\
$\mathrm{~d}_{1.2}$ & 0.6 & 2.223 & 21.02 & 2.72 & 0.51 \\
$\mathrm{e}_{1.2}$ & 0.4 & 2.073 & 26.04 & 4.22 & 0.55 \\
$\mathrm{f}_{1.2}$ & 0.2 & 1.799 & 33.99 & 8.46 & 0.61 \\
$\mathrm{~g}_{1.2}$ & 0 & 1.375 & 49.64 & 0.69 \\
\hline
\end{tabular}

TABLE 2: $\zeta-\psi$ combinations with dimensionless settling energy equivalent to the pure PD control with $\zeta=1$.

\begin{tabular}{lccccc}
\hline Parameter set & $\zeta$ & $\psi$ & Overshoot $(\%)$ & Dimensionless settling time & Dimensionless rise time \\
\hline $\mathrm{a}_{1.0}$ & 1.0 & 0 & 13.39 & 5.41 & 0.73 \\
$\mathrm{~b}_{1.0}$ & 0.833333 & 1.235 & 16.42 & 3.64 & 0.57 \\
$\mathrm{c}_{1.0}$ & 0.666 & 1.461 & 19.69 & 3.27 & 0.58 \\
$\mathrm{~d}_{1.0}$ & 0.5 & 1.457 & 23.89 & 3.14 & 0.61 \\
$\mathrm{e}_{1.0}$ & 0.333 & 1.314 & 30.00 & 4.46 & 0.66 \\
$\mathrm{f}_{1.0}$ & 0.1666 & 1.054 & 40.42 & 7.24 & 0.74 \\
$\mathrm{~g}_{1.0}$ & 0.1 & 0.8962 & 47.43 & 7.94 & 0.79 \\
\hline
\end{tabular}

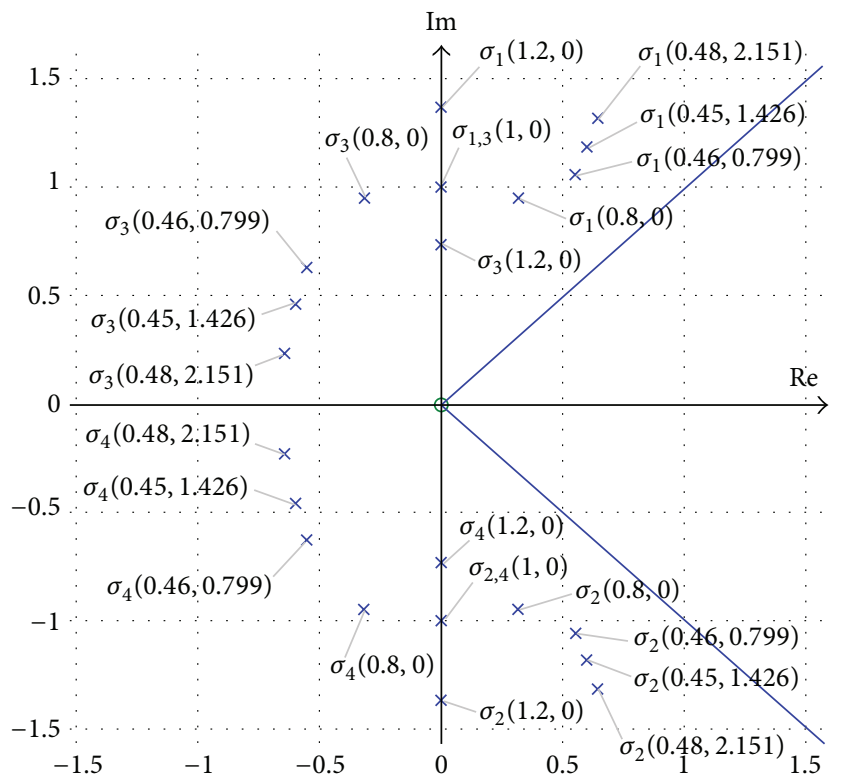

FIgure 10: Pole locations for the six cases of Table 4.

right half-plane; therefore, the system behaviour is always oscillatory.

The minimization of the settling time with the same settling energy is a possible criterion for the combination of the derivative and half-derivative terms which is based on a specific set point time history (the step) and on a secondorder linear systems; however, simulations show that the combination of derivative and half-derivative terms reduces the tracking error also in case of nonlinear systems controlled with different set points [30]. In the following section, the PD and $\mathrm{PDD}^{1 / 2}$ controls characterized by the $\zeta-\psi$ combinations of Table 4 will be experimentally compared with reference to

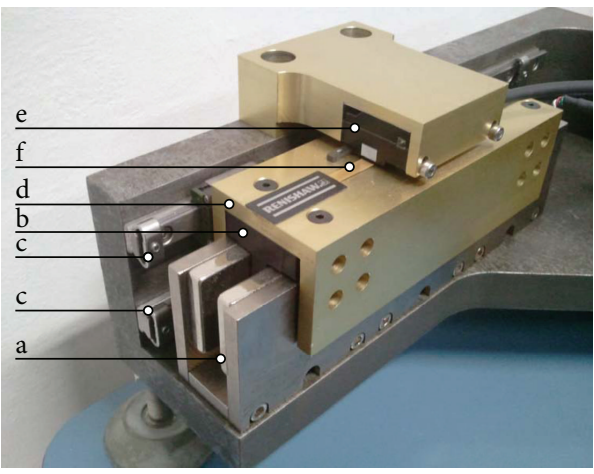

FIGURE 11: Experimental setup.

a micrometric linear axis actuated by a linear brushless DC motor in case of position set point with trapezoidal speed law (constant acceleration, constant speed, and constant deceleration), which is one of the most frequently adopted in usual mechatronic applications.

\section{Experimental Results}

The experimental setup (Figure 11) is a horizontal linear axis actuated by a linear motor Baldor LMCF 04C-HCO, designed for high-precision mechatronic applications that require smooth operation without cogging. It is composed of a stationary magnet track with permanent magnets (Figure 11, a) and a moving coil assembly (Figure 11, b). The motor has a maximum peak force of $173 \mathrm{~N}$ and a maximum continuous force of $58 \mathrm{~N}$. Two parallel high-precision linear bearings THK HSR10 (Figure 11, c) realize the prismatic joint between the base frame and the translating part (Figure 11, d), which has a mass of $0.66 \mathrm{~kg}$. A linear incremental encoder Renishaw RGH22 (Figure 11, e) in combination with a linear tape scale 
TABLE 3: $\zeta-\psi$ combinations with dimensionless settling energy equivalent to the pure PD control with $\zeta=0.8$.

\begin{tabular}{lccccc}
\hline Parameter set & $\zeta$ & $\psi$ & Overshoot $(\%)$ & Dimensionless settling time & Dimensionless rise time \\
\hline $\mathrm{a}_{0.8}$ & 0.8 & 0 & 17.78 & 5.07 & 0.81 \\
$\mathrm{~b}_{0.8}$ & 0.666 & 0.688 & 20.20 & 3.98 & 0.70 \\
$\mathrm{c}_{0.8}$ & 0.5333 & 0.807 & 23.99 & 3.71 & 0.71 \\
$\mathrm{~d}_{0.8}$ & 0.4 & 0.7635 & 29.38 & 5.32 & 0.75 \\
$\mathrm{e}_{0.8}$ & 0.2666 & 0.598 & 38.08 & 8.05 & 0.83 \\
$\mathrm{f}_{0.8}$ & 0.2 & 0.4529 & 45.35 & 9.05 & 0.89 \\
$\mathrm{~g}_{0.8}$ & 0.15 & 0.251 & 55.23 & 15.08 & 0.97 \\
\hline
\end{tabular}

TABLE 4: $\zeta-\psi$ combinations minimizing the dimensionless settling time with dimensionless settling energy equivalent to the pure PD.

\begin{tabular}{lcc}
\hline $\begin{array}{l}\zeta \text { of the PD control with } \\
\text { equivalent settling energy }\end{array}$ & \multicolumn{2}{c}{$\mathrm{PDD}^{1 / 2}$ control minimizing } \\
& $\zeta$ & $\psi$ \\
\hline 1.2 & 0.48 & 2.1510 \\
1 & 0.45 & 1.4266 \\
0.8 & 0.46 & 0.7990 \\
\hline
\end{tabular}

(Figure 11, f) provides a linear resolution of $0.1 \mu \mathrm{m}$ along the $75 \mathrm{~mm}$ travel range.

The control scheme is shown in Figure 12; a motion control unit Baldor NextMove ESB performs the position loop with a sampling time of 1 millisecond, while a motor drive Baldor MicroFlex performs the inner current control loop. In order to compare the $\mathrm{PD}$ and the $\mathrm{PDD}^{1 / 2}$ position controls, the original firmware of the motion controller NextMove ESB has been replaced with a new firmware, programmed in $\mathrm{C}++$, which performs the numerical evaluation of the halfderivative of the position error by means of the discrete-time approximation of (4).

Since the electrical time constant of the motor is $6.6 \cdot 10^{-4} \mathrm{~s}$, the electrical dynamics of the system can be neglected; so the force $F$ applied by the linear motor can be considered proportional to the output of the position loop $i_{r}$, that is, the set-point of the current loop. On the contrary, the friction effects of the linear bearings are not negligible in the system dynamics. In order to model friction during motion, the mechanical dynamics of the linear axis can be described introducing the Coulomb and viscous terms:

$$
m \ddot{x}=-c_{v} \dot{x}-\operatorname{sign}(\dot{x}) c_{s}+k_{t} i,
$$

where $i$ is the motor current, $k_{t}$ is the force constant $(21.6 \mathrm{~N} / \mathrm{A}), c_{v}=0.7 \mathrm{Ns} / \mathrm{m}$ is the viscous friction coefficient, and $c_{s}=0.97 \mathrm{~N}$ is the Coulomb friction; these parameters have been obtained by system identification performing displacement tests on the linear axis.

Using (15), and considering that the current set point is the output of the position loop, the following nonlinear differential equation of the closed-loop system can be obtained:

$m \frac{d^{2}}{d t^{2}} x=-K_{p} e_{x}-K_{d} \frac{d}{d t} e_{x}-K_{h d} \frac{d^{1 / 2}}{d t^{1 / 2}} e_{x}-\operatorname{sign}(\dot{x}) c_{s}-c_{v} \dot{x}$.
The PD and $\mathrm{PDD}^{1 / 2}$ control schemes have been compared by using both the nonlinear model (16) and the experimental layout. In particular, Figure 13 shows the model comparison between the PD and $\mathrm{PDD}^{1 / 2}$ control with a trapezoidal speed law of the position set point characterized by an overall displacement of $10 \mathrm{~mm}$ performed with maximum speed of $100 \mathrm{~mm} / \mathrm{s}$ and constant acceleration and deceleration of $2000 \mathrm{~mm} / \mathrm{s}^{2}$. These speed and acceleration values, which are relatively high with respect to the displacement scale, have been selected to stress the mechanical dynamics and to exalt the differences between the two control strategies.

The gains of the position loop are

(i) for the PD: $K_{p}=1962.7 \mathrm{~N} / \mathrm{m}$ and $K_{d}=71.98 \mathrm{Ns} / \mathrm{m}$ (corresponding to $\zeta=1, \psi=0$ );

(ii) for the $\mathrm{PDD}^{1 / 2}: K_{p}=1962.7 \mathrm{~N} / \mathrm{m}, K_{h d}=$ $379.16 \mathrm{Ns}^{1 / 2} / \mathrm{m}$, and $K_{d}=32.93 \mathrm{Ns} / \mathrm{m}$ (corresponding to $\zeta=0.45, \psi=1.4266$ ).

It is possible to see that the tracking error with the $\mathrm{PDD}^{1 / 2}$ is significantly lower, and the experimental tests are coherent with the simulations; in Figure 14 it is possible to compare the $\mathrm{PD}$ and $\mathrm{PDD}^{1 / 2}$ behaviour both by means of the nonlinear model (16) and by experimental validation. Two different experimental time histories, which correspond to two different executions of the same trajectory, are reported; the comparisons for the other pairs of gain sets of Table 4 lead to similar results; due to the random variability of the microscopic friction phenomena in the linear bearings, different executions of the same trajectory are enclosed within a repeatability range of $40 \mu \mathrm{m}$ for all the gain sets of Table 4, and the maximum difference between the model behaviour and the experimental test is lower than $75 \mu \mathrm{m}$. For example, Figure 15 shows the same comparison of Figure 14 for the gain sets corresponding to the last row of Table 4 (PD: $\left.\zeta=0.8 ; \mathrm{PDD}^{1 / 2}: \zeta=0.46, \psi=0.7990\right)$.

\section{Conclusions}

The application of the $\mathrm{PD}$ and $\mathrm{PDD}^{1 / 2}$ schemes to the position control of a linear axis has been investigated at different levels. First of all, a linear dimensionless model of the closedloop system has been developed, and the system stability is discussed by means of the pole locations in the $\sigma$-plane; such locations indicate that the system is stable if at least one of the derivative and half-derivative gains is greater than zero, 


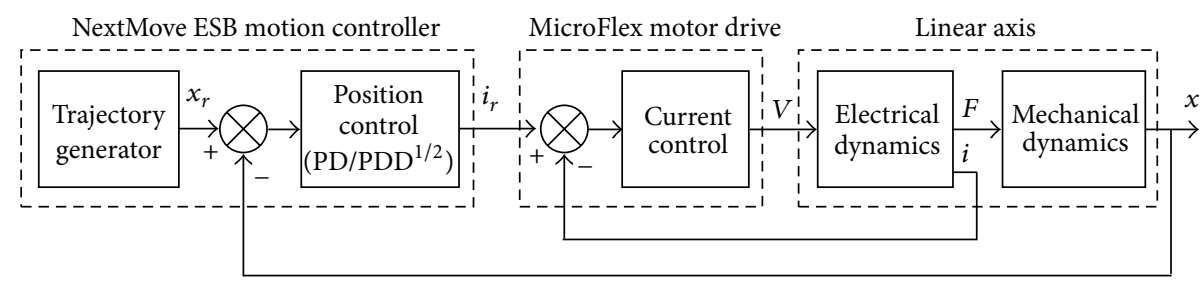

FIGURE 12: Control scheme.

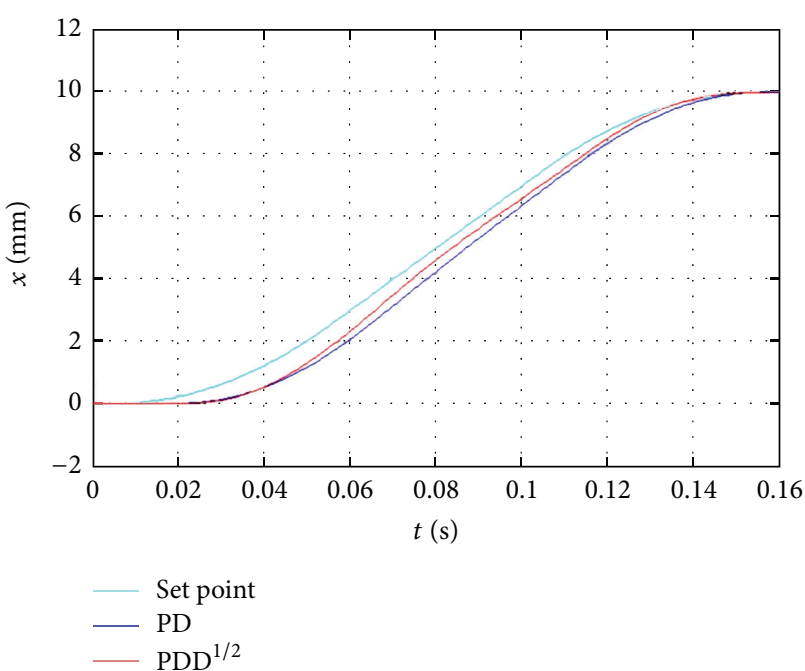

FIGURE 13: PD-PDD ${ }^{1 / 2}$ comparison: displacement in case of set point with trapezoidal speed law $\left(\mathrm{PD}: \zeta=1 ; \mathrm{PDD}^{1 / 2}: \zeta=\right.$ 0.45 , and $\psi=1.4266$ ).

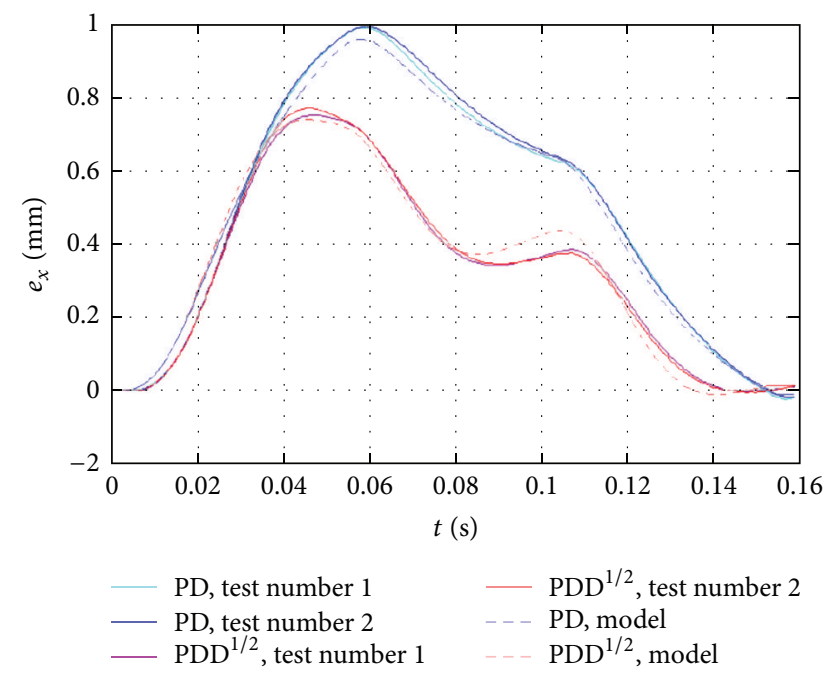

FIGURE 14: PD-PDD ${ }^{1 / 2}$ comparison: tracking error in case of set point with trapezoidal speed law (PD: $\zeta=1 ; \mathrm{PDD}^{1 / 2}: \zeta=$ 0.45 , and $\psi=1.4266)$.

and that increasing values of the half-derivative term induce a more oscillatory behaviour in the time response.

Then, the step response has been considered for different combinations of the derivative and half-derivative terms, characterized by equal the dimensionless settling energy

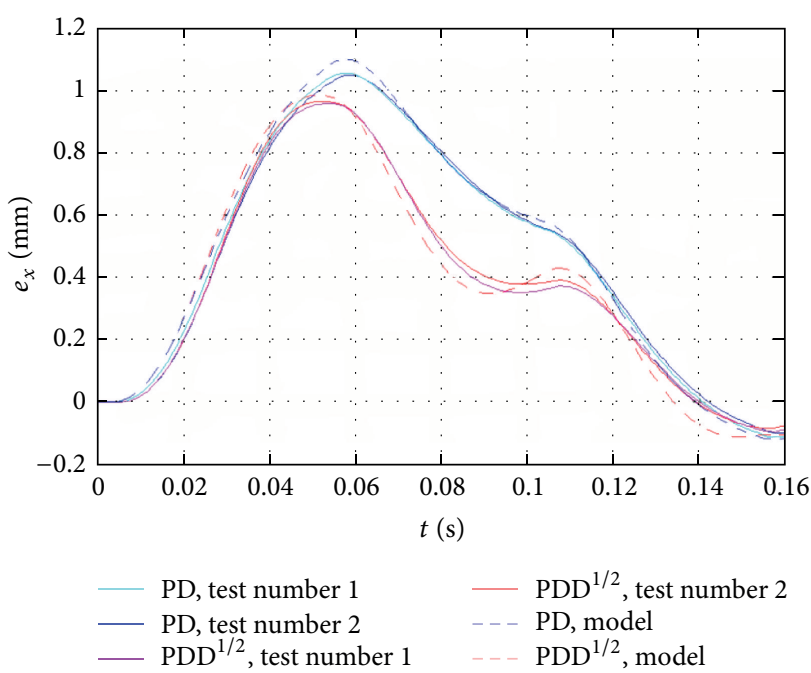

FIGURE 15: PD-PDD ${ }^{1 / 2}$ comparison: tracking error in case of set point with trapezoidal speed law $\left(\mathrm{PD}: \zeta=0.8 ; \mathrm{PDD}^{1 / 2}: \zeta=\right.$ $0.46, \psi=0.7990)$.

(Figures 7-9, Tables 1-3), and for different levels of settling energy, the $\zeta-\psi$ combinations which minimize the settling time are singled out (Table 4 ).

Considering these $\zeta-\psi$ combinations, the PD-PDD ${ }^{1 / 2}$ comparison has been performed experimentally on a micrometric linear axis actuated by a brushless DC motor, with nonnegligible friction phenomena in the linear bearings, and adopting a typical position set point with trapezoidal speed law. The test results show that also in these conditions the combination of the derivative and half-derivative terms allows to reduce significantly the tracking error.

Let us note that the small scale of the experimental setup does not limit the significance of the results, which can be extended to applications in which a linear or rotational axis is actuated with a load which is predominantly inertial and frictional, a case which is quite common in mechatronics; nevertheless, further PD-PDD ${ }^{1 / 2}$ comparisons must be performed considering different types of controlled system, especially if characterized by strong nonlinearity.

In the present work, the analysis has been carried out considering $\zeta-\psi$ combinations with equal settling energy, aiming at minimizing the settling time. Another interesting issue is to minimize the settling energy with equal settling time; this topic is of great importance, for example, for the control of large-scale energy plants, whose efficiency is fundamental also in the transient state. 
From a theoretical point of view, the stability of the $\mathrm{PDD}^{1 / 2}$ control has been analysed only in the case of a second-order plant; further work has to be done to extend the analysis, in particular in the presence of nonlinear friction phenomena which may give rise to limit-cycle behaviour.

For all of these reasons, it is evident that the proposed work is not conclusive; while for the PD control a wide variety of tuning methodologies is already available in the scientific literature, the tuning of the $\mathrm{PDD}^{1 / 2}$ for different plants and operative conditions is a virtually unexplored research filed, with promising applications.

\section{References}

[1] K. B. Oldham and J. Spanier, The Fractional Calculus: Theory and Application of Differentiation and Integration to Arbitrary Order, Academic Press, New York, NY, USA, 1974.

[2] K. S. Miller and B. Ross, An Introduction to the Fractional Calculus and Fractional Differential Equations, John Wiley \& Sons, 1993.

[3] I. Podlubny, Fractional Differential Equations, Academic Press, San Diego, Calif, USA, 1999.

[4] D. Baleanu, "Fractional variational principles in action," Physica Scripta, vol. T136, 2009.

[5] A. Golmankhaneh, A. Golmankhaneh, D. Baleanu, and M. C. Baleanu, "Hamiltonian structure of fractional first order lagrangian," International Journal of Theoretical Physics, vol. 49, no. 2, pp. 365-375, 2010.

[6] S. I. Muslih, O. P. Agrawal, and D. Baleanu, "A fractional Dirac equation and its solution," Journal of Physics A, vol. 43, no. 5, Article ID 055203, 2010.

[7] S. Grimnes and O. G. Martinsen, Bioimpedance and Bioelectricity Basics, Academic Press, San Diego, Calif, USA, 2000.

[8] R. S. Lakes, Viscoelastic Solids, CRC Press, Boca Raton, Fla, USA, 1999.

[9] M. Sasso, G. Palmieri, and D. Amodio, "Application of fractional derivative models in linear viscoelastic problems," Mechanics of Time-Dependent Materials, vol. 15, no. 4, pp. 367-387, 2011.

[10] R. L. Magin, Fractional Calculus in Bioengineering, Begell House, Redding, Conn, USA, 2006.

[11] A. J. Bard and L. R. Faulkner, Electrochemical Methods: Fundamentals and Applications, John Wiley \& Sons, New York, NY, USA, 2nd edition, 2001.

[12] D. Baleanu, A. K. Golmankhaneh, and A. K. Golmankhaneh, "Fractional nambu mechanics," International Journal of Theoretical Physics, vol. 48, no. 4, pp. 1044-1052, 2009.

[13] R. Hilfer, Applications of Fractional Calculus in Physics, World Scientific, 2000.

[14] N. Heymans and I. Podlubny, "Physical interpretation of initial conditions for fractional differential equations with RiemannLiouville fractional derivatives," Rheologica Acta, vol. 45, no. 5, pp. 765-771, 2006.

[15] A. A. Kilbas, H. M. Srivastava, and J. J. Trujillo, Theory and Applications of Fractional Differential Equations, Elsevier, Amsterdam, The Netherlands, 2006.

[16] R. E. Gutiérrez, J. M. Rosário, and J. T. Machado, "Fractional order calculus: basic concepts and engineering applications," Mathematical Problems in Engineering, vol. 2010, Article ID 375858, 19 pages, 2010.
[17] M. Axtell and M. E. Bise, "Fractional calculus applications in control systems," in Proceedings of the IEEE National Aerospace and Electronics Conference (NAECON '90), pp. 563-566, Dayton, Ohio, USA, May 1990.

[18] S. E. Hamamci and M. Koksal, "Calculation of all stabilizing fractional-order PD controllers for integrating time delay systems," Computers and Mathematics with Applications, vol. 59, no. 5, pp. 1621-1629, 2010.

[19] S. E. Hamamci, "Stabilization using fractional-order PI and PID controllers," Nonlinear Dynamics, vol. 51, no. 1-2, pp. 329-343, 2008.

[20] R. Matušů, "Application of fractional order calculus to control theory," International Journal of Mathematical Models and Methods in Applied Sciences, vol. 5, no. 7, pp. 1162-1169, 2011.

[21] I. Podlubny, "Fractional-order systems and $\mathrm{PI}^{\lambda} \mathrm{D}^{\mu}$ controllers," IEEE Transactions on Automatic Control, vol. 44, no. 1, pp. 208213, 1999.

[22] C. Yeroglu and N. Tan, "Note on fractional-order proportionalintegral-differential controller design," IET Control Theory \& Applications, vol. 5, no. 17, pp. 1978-1989, 2012.

[23] G. Ruikun, L. Aiwu, F. Min, G. Lihui, and G. Huanyao, "Study of fractional order $\mathrm{PI}^{\lambda} \mathrm{D}^{\mu}$ controller designing method," in Proceedings of the IEEE Symposium on Robotics and Applications (ISRA '12), pp. 277-281, Kuala Lumpur, Malaysia, June 2012.

[24] P. Ostalczyk and P. Duch, "Closed-Loop system synthesis with the variable-, fractional-Order PID controller," in Proceedings of the 17th International Conference on Methods and Models in Automation and Robotics (MMAR '12), pp. 589-594, Miedzyzdroje, Poland, August 2012.

[25] A. Tepljakov, E. Petlenkov, and J. Belikov, "A flexible MATLAB tool for optimal fractional-order PID controller design subject to specifications," in Proceedings of the 31st Chinese Control Conference (CCC '12), pp. 4698-4703, Hefei, China, July 2012.

[26] W. Guo, Y. Song, L. Zhou, and L. Deng, "A novel model algorithmic controller with fractional order PID structure," in Proceedings of the 10th World Congress on Intelligent Control and Automation (WCICA '12), pp. 2517-2522, Beijing, China, July 2012.

[27] K. Erenturk, "Fractional order $\mathrm{PI}^{\lambda} \mathrm{D}^{\mu}$ and active disturbance rejection control of nonlinear two mass drive system," IEEE Transactions on Industrial Electronics, vol. PP, no. 99, 2012.

[28] M. O. Efe, "Neural network assisted computationally simple $\mathrm{PI}^{\lambda} \mathrm{D}^{\mu}$ control of a quadrotor UAV," IEEE Transactions on Industrial Informatics, vol. 7, no. 2, pp. 354-361, 2011.

[29] R. Duma, P. Dobra, and M. Trusca, "Embedded application of fractional order control," Electronics Letters, vol. 48, no. 24, pp. 1526-1528, 2012.

[30] L. Bruzzone and G. Bozzini, "Fractional-order derivatives and their application to the position control of robots," International Journal of Mechanics and Control, vol. 10, no. 1, pp. 39-44, 2009.

[31] L. Bruzzone and G. Bozzini, "Nondimensional analysis of fractional-order $\mathrm{PDD}^{1 / 2}$ control of purely inertial systems," Journal of Mechatronics and Applications, vol. 2010, Article ID 903420, 10 pages, 2010.

[32] L. Bruzzone and P. Fanghella, "Influence of the half-derivative term on fractional-order control of mechatronic systems," in Proceedings of the 21st International Workshop on Robotics in Alpe-Adria-Danube Region (RAAD '12), pp. 292-298, Naples, Italy, September 2012.

[33] J. A. T. Machado, "Fractional-order derivative approximations in discrete-time control systems," Systems Analysis Modelling Simulation, vol. 34, no. 4, pp. 419-434, 1999. 
[34] Y. Q. Chen, I. Petráš, and D. Xue, "Fractional order control—a tutorial," in Proceedings of the American Control Conference, pp. 1397-1411, St. Louis, Mo, USA, June 2009.

[35] D. Matignon, "Generalized fractional differential and difference equations: stability properties and modelling issues," in Proceedings of the Mathematical Theory of Networks and Systems Symposium, Padova, Italy, 1998.

[36] S. Das, Functional Fractional Calculus for System Identification and Controls, Springer, 2008. 

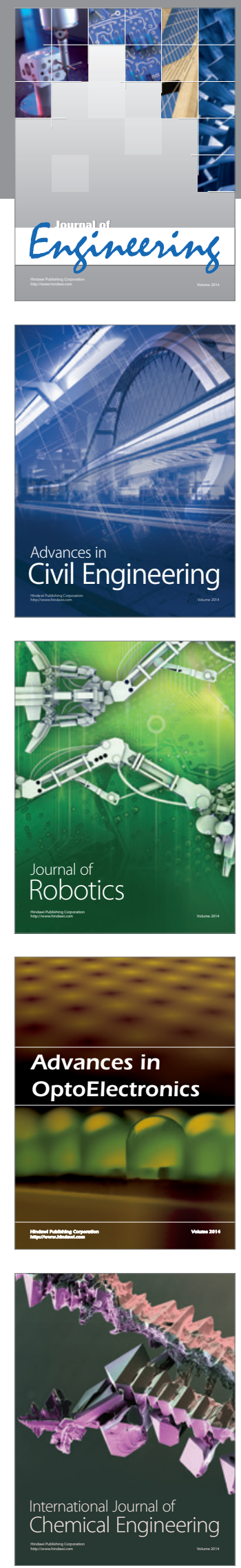

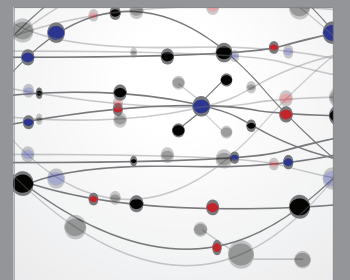

The Scientific World Journal
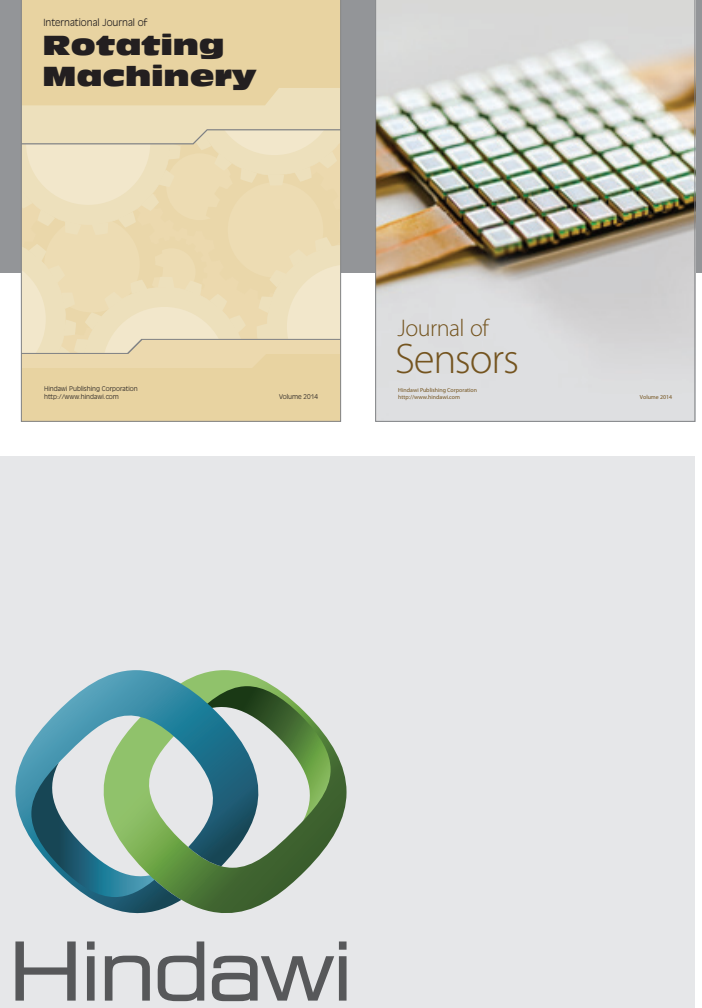

Submit your manuscripts at http://www.hindawi.com
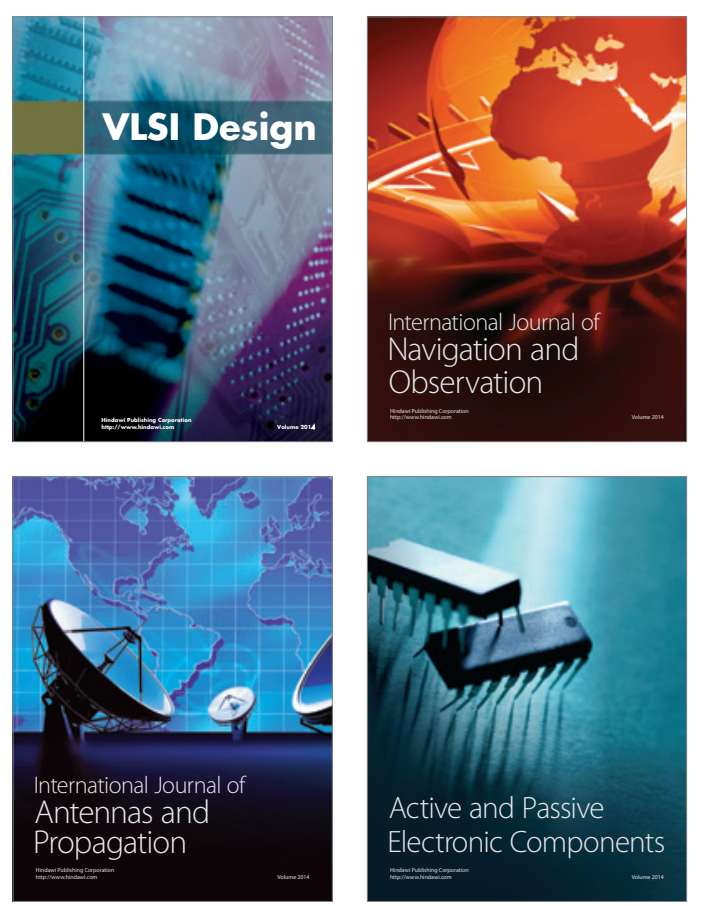
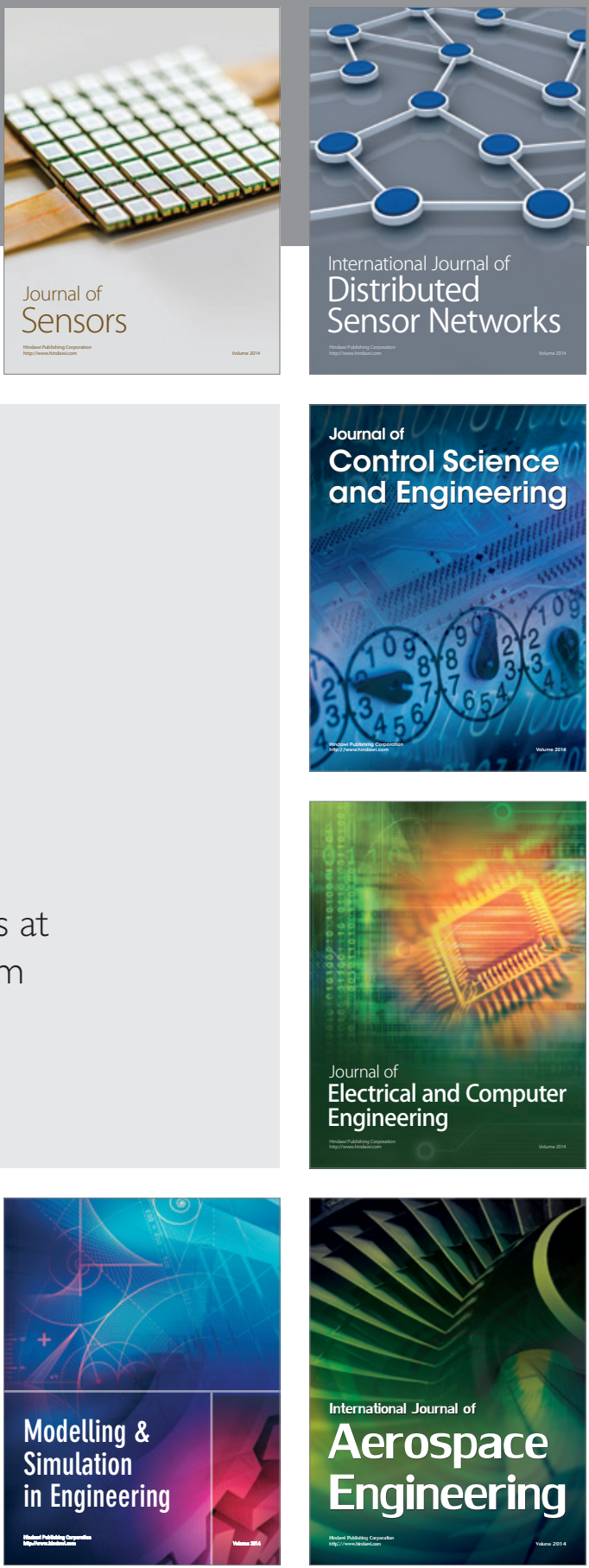

Journal of

Control Science

and Engineering
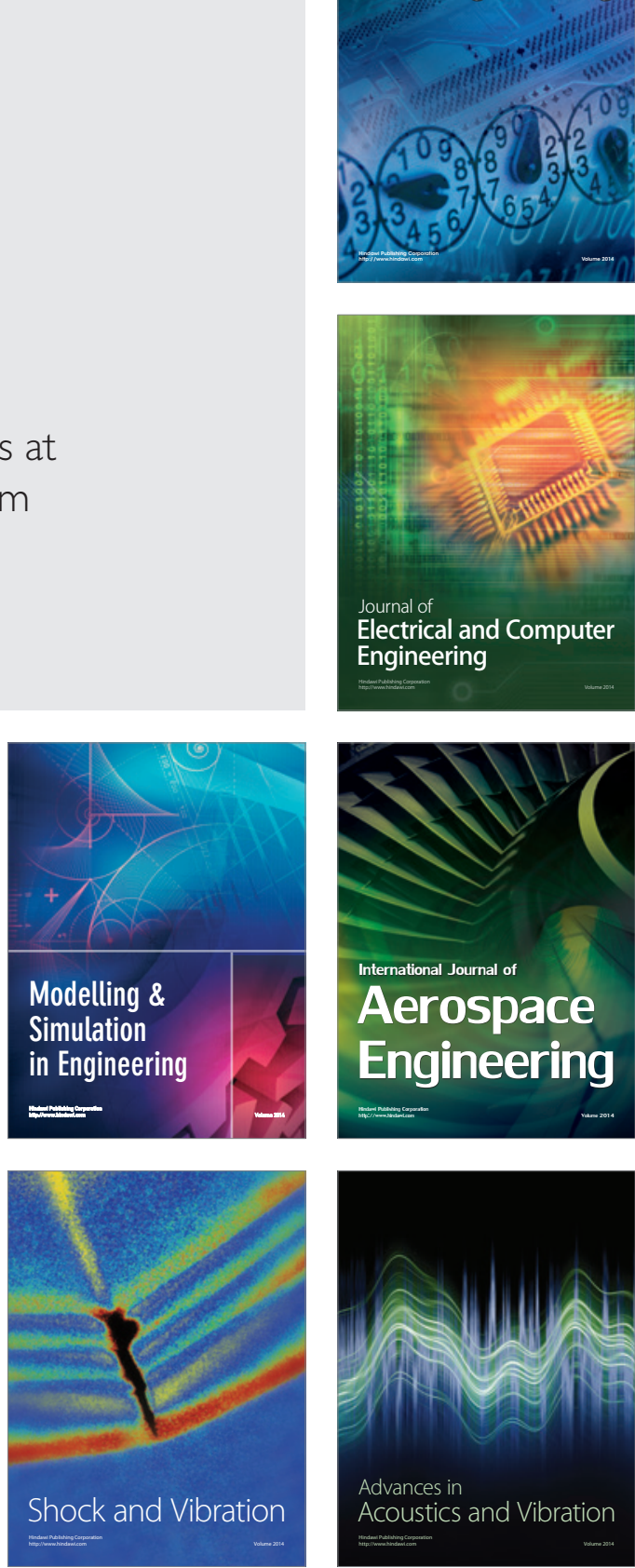